\title{
Genetic Studies in Coloured Sweet Pepper
}

\author{
Amreena Sultan ", Baseerat Afroza, Rizwan Rashid, Afroza Akhter and Azralateef \\ Division of Vegetable Science, Sher-e-Kashmir University of Agricultural Sciences and \\ Technology of Kashmir, Shalimar, Srinagar- 190 025, Jammu and Kashmir (India) \\ *Corresponding author
}

\section{A B S T R A C T}

\begin{tabular}{|l|}
\hline Ke y w o r d s \\
Coloured Sweet \\
pepper, Variability, \\
Heritability, \\
Correlation
\end{tabular}

In the present investigation, fifteen diverse genotypes of coloured capsicum were evaluated in experimental field of Division of Vegetable Science, SKUAST-Kashmir, Shalimar during kharif 2018. Analysis of variance revealed highly significant differences among genotypes for all the traits. Highest genotypic coefficients of variation were depicted by total carotenoids (38.90), fruit yield plant ${ }^{-1}$ (27.93), fruit yield $\operatorname{plot}^{-1}$ (26.99) and fruit weight (20.33). The heritability in broad sense was high above 60 per cent for all traits except quality traits. Genetic advance was found to be low in almost all traits. The genotypic correlation coefficients were higher in magnitude than phenotypic correlation coefficients but similar in direction. Fruit yield plot $^{-1}$ was found to be positively and significantly correlated with plant height, plant spread, number of secondary branches, number of fruits plant ${ }^{-1}$, average fruit weight, fruit length, fruit diameter, flesh thickness, pedicel length, average seed weight fruit ${ }^{-1}$, number of seeds fruit ${ }^{-1}$, fruit yield plant $^{-1}$, seed yield plant ${ }^{-1}$ and seed yield plot $^{-1}$.

\section{Introduction}

The consumption of sweet pepper is on the increase all over the world. It has become a multibillion dollar industry, as well as a part time hobby for home gardeners. Coloured Sweet peppers are gaining popularity day by day due to changing food habits of people, increasing health concerns, and knowledge about benefits of consuming coloured vegetables. The farmers are shifting towards the cultivation of coloured capsicums. However the yield potential and total production of coloured capsicum is low due to the unavailability of high yielding cultivars adaptable to Kashmir valley.
The success of any breeding programme depends on the presence of sufficient variability to pursue effective selection. It is important to assess the relative magnitude of components of variability in order to use such information, together with other selection parameters for improvement of the plant type through adoption of effective breeding method (Johnson et al., 1955; Hanson et al., 1956; William, 1964; Briggs and Knowels, 1967). Burton (1952) suggested that the extent of variability indicates the amenability of a given character for its improvement while the knowledge on the heritability along with genetic advance aid in drawing valuable conclusions for effective selection based on 
phenotypic performance as suggested by Johnson et al., (1955).Correlation studies provide information that selection for one character would result in selection and progress of all traits that are positively and significantly correlated with the selected character.

Correlation coefficient would indicate how much progress might be expected from selecting for two variables simultaneously or in selecting for one variable and expecting to bring about a change in second variable. If significant correlation values are found between yield and other economic traits, considerable improvement could be made through selection.

\section{Materials and Methods}

The present investigation was carried out to generate information regarding variability, heritability and correlation in coloured Sweet pepper genotypes for subsequent use in future breeding programmes. Fifteen diverse genotypes of coloured Capsicum maintained in Division of Vegetable Science were evaluated in Experimental Field, Division of Vegetable Science, SKUAST-K Shalimar. The experiment was laid in RCBD during kharif 2018 with three replications. The observations were recorded on various maturity, yield and yield attributing traits and quality traits. Analysis was done as per standard statistical procedures.

\section{Results and Discussion}

Analysis of variance revealed highly significant differences among genotypes for all the traits under study indicating the presence of sufficient amount of variability in the genotypes. This provides an ample opportunity for selecting suitable genotypes with high mean for all the traits of interest. These results are in accordance with those of Tembhurne and Rao (2013), Abu et al.,
(2013), and Spaldon et al., (2017).

Wide range of variability was observed for most of the traits under study as depicted by range values which is a pre requisite for making improvement through selection. The estimates of phenotypic, genotypic and environmental variances revealed that the magnitude of environmental variances were much smaller than the magnitudes of correspondence genotypic variances indicating that the variations observed were mostly due to genotypes, that is, genetic in nature. Genotypic coefficients of variation were found to be higher than corresponding environmental coefficients of variation. Highest genotypic coefficients of variation were shown by total carotenoids (38.90), fruit yield plant ${ }^{-1}$ (27.93), fruit yield plot $^{-1}$ (26.99) and fruit weight (20.33) indicating that selection for these traits would be effective.

The heritability in broad sense was high above 60 per cent for all traits except quality traits i.e., SSC, vitamin C, total chlorophyll and total carotenoids suggesting that the selection based on phenotype would be more effective and there is every possibility to transmit these traits into off springs. Genetic advance was found to be low in almost all traits except seed yield plot $^{-1}$ (45.40), number of seeds fruit $^{-1}$ (33.10), vitamin C (24.50), total chlorophyll (15.13) and seed yield plant ${ }^{-1}$ (14.53).

High heritability and genetic advance together are helpful in predicting gain under selection. However, when heritability is high and genetic advance is not proportionately high, this situation indicates that non-additive genes with dominance and epistatic effects are controlling the concerned traits. High heritability along with high genetic advance responds to selection better than high heritability and low genetic advance (Table $1-4)$. 
Table.1a Mean square of individual environments for maturity, yield attributing and quality traits in

Coloured Capsicum (Capsicum annuum L. var. grossum Sendt.)

\begin{tabular}{|c|c|c|c|c|c|c|c|c|c|c|c|c|}
\hline \multirow{2}{*}{$\begin{array}{l}\text { Source of } \\
\text { variation }\end{array}$} & \multirow[t]{2}{*}{ d.f } & \multicolumn{11}{|c|}{$\underline{\text { Mean sum of squares }}$} \\
\hline & & $\begin{array}{l}\text { Days to } \\
\text { first } \\
\text { flowering }\end{array}$ & $\begin{array}{l}\text { Days to } \\
\text { first fruit } \\
\text { set }\end{array}$ & $\begin{array}{l}\text { Days to } \\
\text { first fruit } \\
\text { harvest }\end{array}$ & $\begin{array}{l}\text { Plant } \\
\text { height } \\
(\mathrm{cm})\end{array}$ & $\begin{array}{l}\text { Plant } \\
\text { spread } \\
(\mathrm{cm})\end{array}$ & $\begin{array}{l}\text { Number } \\
\text { of } \\
\text { secondary } \\
\text { branches }\end{array}$ & $\begin{array}{l}\text { Number } \\
\text { of fruits } \\
\text { plant }^{-1}\end{array}$ & $\begin{array}{l}\text { Number of } \\
\text { fruits } \\
\text { plant }^{-1}\end{array}$ & $\begin{array}{l}\text { Fruit } \\
\text { Length } \\
(\mathrm{cm})\end{array}$ & $\begin{array}{l}\text { Fruit } \\
\text { diameter } \\
(\mathrm{cm})\end{array}$ & $\begin{array}{l}\text { Flesh } \\
\text { thickness } \\
(\mathbf{m m})\end{array}$ \\
\hline Genotypes & 14 & $11.850 * *$ & $10.660 * *$ & $16.363 * *$ & $94.940 * *$ & $29.521 * *$ & $2.110 * *$ & $22.600 * *$ & $1652.544^{* *}$ & $0.878 * *$ & $0.852 * *$ & $2.071 * *$ \\
\hline Error & 28 & 0.554 & 0.774 & 0.484 & 0.442 & 0.860 & 0.100 & 1.300 & 4.912 & 0.001 & 0.001 & 0.128 \\
\hline
\end{tabular}

Table.1b Mean square of individual environments for maturity, yield attributing and quality traits in Coloured Capsicum (Capsicum annuum L. var. grossum Sendt.)

\begin{tabular}{|c|c|c|c|c|c|c|c|c|c|c|c|c|}
\hline \multirow{2}{*}{$\begin{array}{l}\text { Source of } \\
\text { variation }\end{array}$} & \multirow[t]{2}{*}{ d.f } & \multicolumn{11}{|c|}{$\underline{\text { Mean sum of squares }}$} \\
\hline & & $\begin{array}{l}\text { Pedicel } \\
\text { length } \\
(\mathrm{cm})\end{array}$ & $\begin{array}{l}\text { Average } \\
\text { seed } \\
\text { weight } \\
\text { (g) }\end{array}$ & $\begin{array}{l}\text { Number of } \\
\text { seeds fruit } \\
1\end{array}$ & $\begin{array}{l}\text { Average } \\
\text { fruit } \\
\text { yield } \\
\text { plant }^{-1} \\
\text { (kg) }\end{array}$ & $\begin{array}{l}\text { Average } \\
\text { fruit } \\
\text { yield } \\
\text { plot }^{-1} \\
\text { (kg) }\end{array}$ & $\begin{array}{l}\text { Seed } \\
\text { yield } \\
\text { plant }^{-1}(g)\end{array}$ & $\begin{array}{l}\text { Seed yield } \\
\operatorname{plot}^{-1}(g)\end{array}$ & $\begin{array}{l}\text { Soluble } \\
\text { solid } \\
\text { content } \\
\text { SSC } \\
\left({ }^{\circ} \text { B }\right)\end{array}$ & $\begin{array}{l}\text { Vitamin C } \\
(\mathrm{mg} / \mathbf{1 0 0 g})\end{array}$ & $\begin{array}{l}\text { Total } \\
\text { chlorophyll } \\
\text { (mg/100g) }\end{array}$ & $\begin{array}{l}\text { Total } \\
\text { carotenoid } \\
\text { (mg/100g) }\end{array}$ \\
\hline Genotypes & 14 & $0.747 * *$ & $1.264 * *$ & $5502.519 * *$ & $0.298 * *$ & $30.190 * *$ & $317.508^{* *}$ & $31788.956 * *$ & $1.538 * *$ & $1873.355^{* *}$ & $754.963 * *$ & $0.483 * *$ \\
\hline Error & 28 & 0.045 & 0.001 & 6.542 & 0.001 & 0.162 & 3.211 & 317.208 & 0.010 & 1.203 & 0.988 & 0.004 \\
\hline
\end{tabular}


Table.2 Variability parameters for maturity, yield attributing and quality traits in Coloured Capsicum (Capsicum annuum L. var. grossum Sendt.)

\begin{tabular}{|c|c|c|c|c|c|c|c|c|c|}
\hline S.No & Traits & Mean & Range & $\begin{array}{l}\text { Phenotypic } \\
\text { coefficient } \\
\text { of variance } \\
(\mathrm{PCV}) \%\end{array}$ & $\begin{array}{l}\text { Genotypic } \\
\text { coefficient } \\
\text { of variance } \\
\text { (GCV) } \%\end{array}$ & $\begin{array}{l}\text { Phenotypic } \\
\text { variance } \\
\left(\sigma^{2} p\right)\end{array}$ & $\begin{array}{l}\text { Genotypic } \\
\text { variance } \\
\left(\sigma^{2} g\right)\end{array}$ & $\begin{array}{l}\text { Heritability } \\
\text { in } \\
\text { broad sense } \\
(\%)\end{array}$ & $\begin{array}{l}\text { Genetic } \\
\text { advance } \\
(\%)\end{array}$ \\
\hline 1. & Days to first flowering & 31.30 & $28.00-34.82$ & 7.38 & 6.23 & 5.34 & 3.81 & 71.35 & 3.40 \\
\hline 2. & Days to first fruit set & 36.68 & $33.64-39.06$ & 5.47 & 4.80 & 4.03 & 3.10 & 76.92 & 3.17 \\
\hline 3. & Days to first harvest & 52.48 & $49.86-55.53$ & 4.36 & 3.39 & 5.25 & 3.17 & 60.38 & 2.85 \\
\hline 4. & Plant height (cm) & 46.15 & $38.99-52.60$ & 12.39 & 10.27 & 32.73 & 22.49 & 68.71 & 8.09 \\
\hline 5. & Plant spread (cm) & 41.55 & $38.89-45.27$ & 7.15 & 6.28 & 8.83 & 6.82 & 77.24 & 4.73 \\
\hline 6. & No. of sec. branches plant ${ }^{-1}$ & 7.34 & $6.64-8.08$ & 11.49 & 9.43 & 0.71 & 0.48 & 67.60 & 1.17 \\
\hline 7. & Number of fruits plant ${ }^{-1}$ & 11.00 & $8.75-13.77$ & 23.57 & 19.81 & 6.73 & 4.75 & 70.58 & 3.77 \\
\hline 8. & Average Fruit weight (g) & 90.93 & $77.16-106.05$ & 25.61 & 20.33 & 542.30 & 341.77 & 63.02 & 2.81 \\
\hline 9. & Fruit length $(\mathrm{cm})$ & 6.96 & $6.49-7.47$ & 7.92 & 6.26 & 0.30 & 0.19 & 63.33 & 0.71 \\
\hline 10. & Fruit diameter (cm) & 6.88 & $6.49-7.28$ & 6.00 & 5.24 & 0.17 & 0.13 & 76.49 & 0.65 \\
\hline 11. & Flesh thickness (mm) & 5.29 & $4.66-5.86$ & 14.68 & 11.80 & 0.60 & 0.39 & 65.00 & 1.04 \\
\hline 12. & Pedicel length $(\mathrm{cm})$ & 3.07 & $2.44-3.54$ & 15.30 & 13.43 & 0.22 & 0.17 & 77.29 & 0.80 \\
\hline 13. & Average seed weight fruit $^{-1}$ (g) & 4.17 & $3.87-4.62$ & 12.72 & 10.98 & 0.28 & 0.21 & 75.00 & 0.82 \\
\hline 14. & Number of seeds fruit ${ }^{-1}$ & 250.85 & $230.86-262.91$ & 9.43 & 7.76 & 559.76 & 379.86 & 67.86 & 33.10 \\
\hline 15. & Average fruit yield plant ${ }^{-1}(\mathrm{~kg})$ & 0.98 & $0.72-1.27$ & 31.14 & 26.99 & 0.09 & 0.07 & 77.78 & 0.48 \\
\hline 16. & Average fruit yield plot ${ }^{-1}(\mathrm{~kg})$ & 9.85 & $7.27-12.80$ & 31.25 & 27.93 & 9.48 & 7.57 & 79.85 & 5.10 \\
\hline 17. & Seed yield plant ${ }^{-1}(\mathrm{~g})$ & 44.80 & $32.73-54.43$ & 22.54 & 18.76 & 102.03 & 70.64 & 69.23 & 14.53 \\
\hline 18. & Seed yield plot ${ }^{-1}(\mathrm{~g})$ & 448.09 & $327.36-544.33$ & 22.54 & 18.72 & 1020.00 & 704.00 & 69.00 & 45.40 \\
\hline 19. & SSC $\left({ }^{\circ}\right.$ Brix $)$ & 3.80 & $3.08-4.48$ & 17.81 & 13.15 & 0.46 & 0.25 & 54.35 & 0.80 \\
\hline 20. & Vitamin-C (mg/100g) & 151.72 & $125.90-168.80$ & 15.06 & 10.86 & 522.33 & 271.65 & 52.00 & 24.50 \\
\hline 21. & Total chlorophyll (mg/100g) & 73.56 & $59.69-81.14$ & 17.83 & 13.34 & 172.17 & 96.41 & 56.00 & 15.13 \\
\hline 22. & Total carotenoids (mg/100g) & 0.68 & $0.36-1.01$ & 55.45 & 38.90 & 0.14 & 0.07 & 50.00 & 0.4 \\
\hline
\end{tabular}


Table.4 Estimates of genotypic (above diagonal) correlation coefficients among different traits in coloured capsicum (Capsicum annuum var. grossum Sendt.)

\begin{tabular}{|c|c|c|c|c|c|c|c|c|c|c|c|c|c|c|c|c|c|c|}
\hline & DFF & DFS & DFH & PH & PS & NSB & NFP & AFW & FL & FD & FT & $\mathbf{P L}$ & ASW & NSF & FYP & FYPP & SYP & SYPP \\
\hline DFF & $\begin{array}{l}1.00 \\
0\end{array}$ & $0.906^{* *}$ & $0.928^{* * *}$ & $-\overline{0.924 * *}$ & -0.198 & $-0.280^{*}$ & - & -0.070 & $-\overline{0.383 * *}$ & 0.040 & $0.403^{* *}$ & $\overline{0.056^{* * *}}$ & - & -0.075 & $\overline{0.616^{* *}}$ & $\overline{0.617^{* *}}$ & -0.116 & -0.116 \\
\hline DFS & & 1.000 & $0.948 * *$ & $-\overline{0.609 * *}$ & -0.176 & -0.209 & $\overline{0}-977^{* *}$ & -0.053 & $-0.202^{*}$ & 0.091 & 0.097 & -0.021 & $-0.215^{*}$ & -0.140 & $-\overline{0.629 * *}$ & $-\overline{0.630 * *}$ & -0.027 & -0.027 \\
\hline DFH & & & 1.000 & $-\overline{0.830 * *}$ & -0.170 & -0.208 & $\overline{-}^{0.993^{* *}}$ & -0.091 & $-0.246^{*}$ & 0.030 & 0.070 & -0.041 & $0.640 * *$ & -0.097 & $\overline{0.644 * *}$ & $-\overline{0.644 * *}$ & $-0.234 *$ & $-0.234 *$ \\
\hline PH & & & & 1.000 & $0.656^{* *}$ & $0.617 * *$ & $0.423 * *$ & $0.230^{*}$ & $0.536^{* *}$ & 0.038 & $-\overline{0.607 * *}$ & 0.112 & 0.001 & 0.080 & $0.882 * *$ & $0.880^{* *}$ & 0.893 ** & $0.894 * *$ \\
\hline PS & & & & & 1.000 & -0.183 & $0.235^{*}$ & 0.061 & 0.065 & 0.182 & -0.174 & 0.115 & 0.088 & 0.044 & $0.620^{* * *}$ & 0.061 & $0.537^{* * *}$ & $0.537 * *$ \\
\hline NSB & & & & & & 1.000 & $0.374 * *$ & -0.198 & $\overline{-}-490^{* *}$ & -0.178 & -0.199 & 0.195 & $\overline{-}-363^{* *}$ & 0.046 & 0.551 & 0.056 & 0.205 & 0.205 \\
\hline NFP & & & & & & & 1.000 & $0.406^{* *}$ & $-0.313^{*}$ & $-0.534 *$ & $-0.207^{*}$ & 0.013 & -0.036 & 0.054 & $0.806^{* * *}$ & $0.809 * *$ & $0.846 * *$ & $0.846^{* * *}$ \\
\hline AFW & & & & & & & & 1.000 & $0.668^{* *}$ & $0.284 *$ & $0.418^{* *}$ & $0.900 * *$ & $0.243 *$ & $0.813 *$ & 0.130 & $0.271^{*}$ & $0.267 *$ & $0.266 * *$ \\
\hline FL & & & & & & & & & 1.000 & $0.728 * *$ & 0.133 & $0.540^{* * *}$ & $0.591 * *$ & 0.163 & 0.209 & $0.207 *$ & 0.043 & 0.044 \\
\hline FD & & & & & & & & & & 1.000 & 0.151 & $0.551 * *$ & 0.176 & 0.166 & $0.588 * *$ & $0.584^{* *}$ & $0.360 * *$ & $0.359 * *$ \\
\hline FT & & & & & & & & & & & 1.000 & -0.181 & -0.123 & -0.163 & $0.708^{* *}$ & 0.078 & 0.007 & 0.007 \\
\hline PL & & & & & & & & & & & & 1.000 & 0.076 & 0.183 & $0.301 *$ & 0.030 & $0.598^{* *}$ & $0.599 * *$ \\
\hline ASW & & & & & & & & & & & & & 1.000 & $0.245^{*}$ & $0.408^{* * *}$ & $0.408^{* *}$ & $0.508 * *$ & $0.508^{* *}$ \\
\hline NSF & & & & & & & & & & & & & & 1.000 & $0.262^{*}$ & 0.026 & $0.350^{* * *}$ & $0.351 * *$ \\
\hline FYP & & & & & & & & & & & & & & & 1.000 & $1.000^{* *}$ & $0.892 * *$ & $0.892 * *$ \\
\hline FYPP & & & & & & & & & & & & & & & & 1.000 & $0.894 * *$ & $0.895^{* *}$ \\
\hline SYP & & & & & & & & & & & & & & & & & 1.000 & $1.000 * *$ \\
\hline SYPP & & & & & & & & & & & & & & & & & & 1.000 \\
\hline
\end{tabular}

*, ** significant at $5 \%$ and $1 *$ respectively

DFF: Days to first flowering

NFP: Number of fruits plant ${ }^{-1}$

ASW: Av. seed weight fruit $^{1}$
DFS: Days to first fruit set AFW: Average fruit weight (g) NSF: No. of seeds fruit ${ }^{-1}$
DFH: Days to first harvest

FL: Fruit length $(\mathrm{cm})$

FYP: Fruit yield plant ${ }^{-1}(\mathrm{~kg})$
PH: Plant height (cm)

FD: Fruit diameter $(\mathrm{cm})$

FYPP: Fruit yield plot $^{-1}(\mathrm{~kg})$
PS: Plant spread (cm)

FT: Flesh thickness (mm) SYP: Seed yield plant- $^{1}$
NSB: No. of secondary branches plant $^{-1}$

PL: Pedicel length (cm)

SYPP: Seed yield plot $^{-1}(\mathrm{~g})$ 
Similar results on variability, variances and heritability and genetic advance have also been reported by Mishra et al., (2001), Sharma and Sharma (2006), Kumar et al., (2010), Prema et al., (2010), Ramya et al., (2016), Najeema et al., (2018), etc.

In the present study, the genotypic correlation coefficients were higher in magnitude than corresponding phenotypic correlation coefficients but similar in direction. The maturity traits viz. days to flowering, days to first fruit set and days to first harvest were positively and significantly correlated with each other but negatively and significantly correlated with plant height, number of fruits plant $^{-1}$, fruit length, pedicel length, average seed weight fruit ${ }^{-1}$, fruit yield plant $^{-1}$ and fruit yield plot $^{-1}$. The most economic trait viz. fruit yield plot $^{-1}$ was found to be positively and significantly correlated with plant height, plant spread, number of secondary branches, number of fruits plant ${ }^{-1}$, average fruit weight, fruit length, fruit diameter, flesh thickness, pedicel length, average seed weight fruit $^{-1}$, number of seeds fruit ${ }^{-1}$, fruit yield plant ${ }^{-1}$, seed yield plant ${ }^{-1}$ and seed yield plot $^{-1}$ thereby indicating that the selection for these traits would automatically bring about improvement in yield. Several research workers viz. Aliyu et al., (2000), Feipeng and Huang (2004), Bindal et al., (2005), Lahbib et al., (2012), etc have reported significant correlations between different traits in sweet pepper. Correlation studies between yield and its components are of great value in planning and evaluating breeding populations. The knowledge of degree of correlation of yield or any other character of interest with its component traits is important in the construction of selection indices to enable effective direct selection.

\section{References}

Abu, Ngozi., E., Uguru, M.I. and Obi, I. U. 2013. Genotypic and correlation among quantitative characters in genotypes of aromatic pepper grown over years. African Journal of Biotechnology 12(20): 2792-2801.

Aliyu L., Ahmed., M. K. and Magaji, M. D. 2000. Correlation and multiple regression analysis between morphological traits and components of yield in pepper (Capsicum annuum L.). Crop Research Hisar 19(2): 318-323.

Bindal, A. K., Sonia Sood and Smita Kaul. 2005. Correlation and path coefficient analysis studies in capsicum (Capsicum annuum L.). Udyanika Journal of Horticultural Science 11(2): 113-116.

Briggs, F.N. and Knowels, P.F. 1967. Introduction to plant breeding. Reinhold Publishing Corporation, New York/London.

Burton, G.W. and Devane, C.H. 1953. Estimating heritability in tall fescue (Festuca arundinacea) from replicated clonal material. Agronomy Journal, 45: 478-481.

Feipeng Wei and Yuji Huang. 2004. Correlation and path analysis of the main agronomic traits of sweet pepper. Chinese Journal of Tropical Crops 25(4): 50-53.

Hanson, C.H., Robinson, H.F. and Comstock, R.E. 1956. Biometrical studies of yield in segregating population of Korean lespedezd. Agronomy Journal 48(6): 268-272.

Johnson, H.W., Robinson, H.F. and Comstock, R.E. 1955. Estimates of genetic and environmental variability in soyabean. Agronomy Journal 47: 314318.

Kumar, S., Rattan, P., Sharma, J.P. and Gupta, R.K. 2010. Genetic variation and interrelationship studies in chilli (Capsicum annuum L.). Journal of Research SKUAST-J 9(1): 132-136.

Lahbib Karima, BnejdiFethi and Gazzah Mohamed EI. 2012. Genetic diversity 
evaluation of pepper (Capsicum annuиm L.) in Tunisia based on morphological traits. African Journal of Agricultural Research 7(23): 3413-3417.

Mishra, A., Sahu, G.S. and Mishra, P.K.2001. Variability in fruit traits of chilli (Capsicumannuum L.). The Orrisa Journal of Horticulture 29 (2): 107-109.

Najeema. M. H., Revanappa., H. P. Hadimani and I. B. Biradar. 2018. Evaluation of Cherry tomato (Solanumlycopersicum var. cerasiforme) genotypes for yield and quality traits. Int. J. Curr. Microbiol. App. Sci. 7(6): 2433-2439.

Prema.G., Indiresh, K.M. and Santhosha, H.M. 2010. Evaluation of cherry tomat0 (Solanum lycopersicum var. Cerasiforme) genotypes for growth, yield and quality traits. The Asian Journal of Horticulture. 6:181-184.

Ramya, R., Ananthan, M. and Krishnamoorthy, V. 2016. Evaluation of cherry tomato (Solanumlycopersicum L. var. cerasiforme Mill.) genotypes for yield and quality traits. Asian Journal of Horticulture 11 (2): 329-334.

Sharma, A., and Sharma, S. 2006. Variation studies for bell pepper (Capsicum annuит (L.) Sendt. var.grossum) improvement under cold desert conditions of North-Western Himalayas. Indian Journal of Genetics and Plant Breeding 66(4): 357-358.

Spaldon. S., Samnotra, R.K.,Rinchan, D. and Choudhary, D. 2017. Stability analysis and genotype $\times$ environment interaction of quality traits in tomato (Solanum lycopersicum L.). Int.J. Curr. Microbiol. App.Sci. 6 (2): 1506-1515.

Tembhurne, B. V. and Rao, S. K. 2013. Stability analysis in chilli (Capsicum annumL.)Journal of Spices and Aromatic Crops 22(2): 154-164.

Williams, W. 1964. Genetical priniciples and plant breeding. Blackwell Scientific Publication, Oxford, London.

\section{How to cite this article:}

Amreena Sultan, Baseerat Afroza, Rizwan Rashid, Afroza Akhter and Azralateef. 2020. Genetic Studies in Coloured Sweet Pepper. Int.J.Curr.Microbiol.App.Sci. 9(09): 2309-2315. doi: https://doi.org/10.20546/ijcmas.2020.909.288 